\title{
Analysis of Two Infrared Bands of $\mathrm{CH}_{2} \mathrm{D}_{2}$
}

\author{
Wm. Bruce Olson, Harry C. Allen, Jr., and Earle K. Plyler
}

(September 19, 1962)

\begin{abstract}
Two infrared absorption bands of $\mathrm{CH}_{2} \mathrm{D}_{2}$ have been analyzed in the semirigid rotor approximation. These are the A-type band at $2671.67 \mathrm{~cm}^{-1}$ and the C-type band at 4425.61 $\mathrm{cm}^{-1}$. The A-type band has previously been assigned as $\nu_{3}+\nu_{9}$, and the C-type band is tentatively assigned as $\nu_{3}+\nu_{6}$. The upper state of the A-type band is perturbed presumably by the close lying level $2 \nu_{5}$. This interaction has not been investigated. The following values were found for the rotational constants of the ground vibrational state: $A_{0}=4.303$ $\mathrm{cm}^{-1}, \quad B_{0}=3.504 \mathrm{~cm}^{-1}, C_{0}=3.049 \mathrm{~cm}^{-1}$.
\end{abstract}

\section{Introduction}

The rotational-vibrational spectra of all the deuterated species of methane except $\mathrm{CH}_{2} \mathrm{D}_{2}$ have been well investigated [1]. ${ }^{1}$

Methane and $\mathrm{CD}_{4}$ are spherical tops while $\mathrm{CH}_{3} \mathrm{D}$ and $\mathrm{CD}_{3} \mathrm{H}$ are symmetric rotors; on the other hand $\mathrm{CH}_{2} \mathrm{D}_{2}$ is an asymmetric rotor. It seemed of interest to determine the rotational constants of $\mathrm{CH}_{2} \mathrm{D}_{2}$ in order to have a set of constants for each of the species. Fortunately, high resolution spectra could be obtained for both an A-type and a C-type band for this molecule. As recently discussed [2], this is sufficient data to enable a good determination of the ground state constants using the complementary $\Delta F_{2}^{\prime \prime \prime}$ values obtained from the two bands. These two bands have been analyzed in the semirigid approximation to yield good values of the ground state constants. Unfortunately a perturbation in the excited state of the A-type band introduces an uncertainty in the effective constants for this band that is larger than can be justified for the precision of the data. Only transitions involving levels with low values of the rotational quantum numbers have been used in the analysis in order to minimize the effect of centrifugal distortion.

\section{Experimental Procedure}

The spectra were recorded with the grating instrument of the Infrared Spectroscopy Section [3] using a 10,000 lines/in. grating with a ruled area of about $6 \times 8$ in. A cooled PbS photoconductive cell was used as the detector.

Both the A- and C-type bands were recorded using a multiple reflection cell with a total path length of $6 \mathrm{~m}$, filled with $\mathrm{CH}_{2} \mathrm{D}_{2}$ to a pressure of $2 \mathrm{~cm}$ of $\mathrm{Hg}$. The C-type band was observed in the second order of the grating and the A-type band in the first. The $\mathrm{CH}_{2} \mathrm{D}_{2}$ obtained from Merck \& Co., Ltd., had a stated minimum purity of $98 \%$.

\footnotetext{
* This work was supported in part by the Division of Chemistry, Atomic Energy Commission.

1 Figures in brackets indicate the literature references at the end of this paper.
}

The wavelengths of the lines were measured using higher order infrared emission lines of the rare gases as standards, and interpolating between them through the use of the fringes of a Fabry-Perot interferometer as previously described [4].

\section{Theory}

Preliminary to the actual analysis of the spectra the mean square values of the angular momenta about the three principal axes of inertia and the intensities were calculated.

Assuming tetrahedral geometry and identical bond lengths of $1.094 \mathrm{~A}$ for $\mathrm{CH}_{2} \mathrm{D}_{2}$ the moments of inertia, reciprocal moments in $\mathrm{cm}^{-1}$ units, and the asymmetry parameter $\kappa$ were obtained.

For the calculated value of $k=-0.27, \alpha, \beta$, and $\gamma$, as defined by Allen [2], were obtained by linear interpolation in published tables [5] of $E(\kappa)$ for each energy level.

$\alpha, \beta$, and $\gamma$ may be shown to be identical with $\left\langle P_{z}^{2}\right\rangle,\left\langle P_{x}^{2}\right\rangle$, and $\left\langle P_{y}^{2}\right\rangle$, respectively, in an $I^{r}$ representation [6]. The energy of a rotational level in a given vibrational state, neglecting centrifugal distortion, may be written as [2]:

$$
E\left(J_{K_{-1}, K_{1}}\right)=\alpha A_{v}+\beta B_{v}+\gamma C_{v},
$$

and the difference between two rotational levels in the same vibrational state as:

$$
\Delta F=\Delta \alpha A_{v}+\Delta \beta B_{v}+\Delta \gamma C_{v} .
$$

The $\Delta \alpha$ 's, $\Delta \beta$ 's and $\Delta \gamma^{\prime}$ 's for each of the $\Delta F_{2}$ 's observable from strong transitions in the $\mathrm{A}$ - and C-type band were calculated.

The strong transitions for the A-type band are those which satisfy the selection rules:

$$
\Delta J=0, \pm 1 \quad \Delta K_{-1}=0 \quad \Delta K_{1}= \pm 1,
$$

and for the C-type band the strong transitions are those for which

$$
\Delta J=0, \pm 1 \quad \Delta K_{-1}= \pm 1 \quad \Delta K_{1}==0 .
$$


The relative intensities of the transitions were calculated by combining the Boltzmann factor, calculated using the estimated moments of inertia, and nuclear spin statistics with the appropriate line strengths from published tables [7].

The nuclear spins of the equivalent pairs of hydrogen and deuterium atoms in $\mathrm{CH}_{2} \mathrm{D}_{2}$ give rise to degeneracies of the rotational levels. For the ground vibrational state the statistical weight factors are 15 for the symmetric (A) rotational levels and 21 for the antisymmetric (B) levels.

The A-type band at $2672 \mathrm{~cm}^{-1}$ has been previously observed by Wilmshurst and Bernstein [8] who assigned it to the combination $\nu_{3}+\nu_{9}$. As the observed band type is consistent with this assignment and there should be no other bands of this type near $2670 \mathrm{~cm}^{-1}$, there appears to be no reason to doubt the assignment.
The C-type band at $4425 \mathrm{~cm}^{-1}$ can best be assigned at $\nu_{3}+\nu_{6}$ as this seems to be the only combination which would give a C-type band in the region $4400-4500 \mathrm{~cm}^{-1}$.

\section{Analysis}

As each of the bands was isolated from others of comparable intensity, initial assignments, with the aid of the calculated intensities, could be made by inspection. From the initial assignments $\Delta F_{2}$ values were obtained, and with the calculated $\Delta \alpha$ 's, $\Delta \beta$ 's, and $\Delta \gamma$ 's were used to refine the values of $A, B$, and $C$ in the ground and upper vibrational states. From these values of $A, B$, and $C, \alpha$ 's, $\beta$ 's, and $\gamma$ 's pertinent to the value of $\kappa$ in each vibrational state were calculated as before.

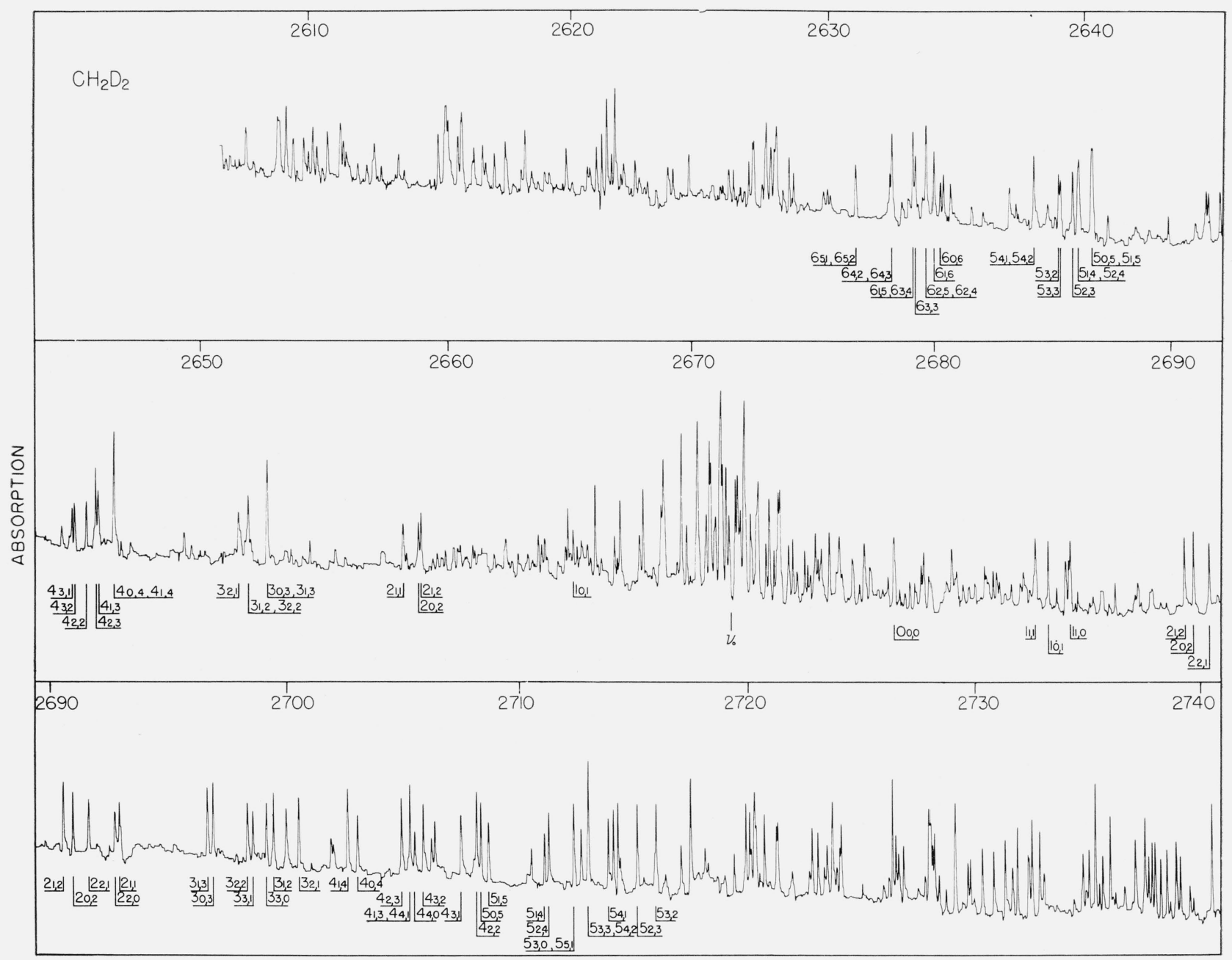

WAVE NUMBER, $\mathrm{cm}^{-1}$

Figure 1. The A-type band of $\mathrm{CH}_{2} \mathrm{D}_{2}$ at $2672 \mathrm{~cm}^{-1}$.

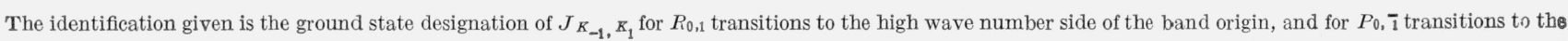
low wave number side of the band origin. 
A trial spectrum was then calculated from the expression:

$$
\nu=\nu_{0}+\alpha^{\prime} A_{v}+\beta^{\prime} B_{v}+\gamma^{\prime} C_{v}-\alpha A_{0}-\beta B_{0}-\gamma C_{0} .
$$

From the trial spectrum and intensities more transitions could be assigned, enabling further refinement of the reciprocal moments of inertia.

While this iterative procedure worked well with the C-type band, Coriolis perturbations in the A-type band caused some difficulty in definitely locating some of the transitions.

The lowest observably perturbed level in the $\nu_{3}+\nu_{9}$ vibrational state is the $4_{13}$ level which is pushed down by $0.24 \mathrm{~cm}^{-1}$. For $J=5$, the levels $5_{05}, 5_{14}$, and $5_{23}$ are all perturbed, and for $J=6$ over half of the levels are perturbed.
This perturbation has not been investigated in detail. It probably arises through interaction with the vibrational state $2 \nu_{5}$, the fundamental of which is theoretically inactive in the infrared, but appears to have been observed at $1329 \mathrm{~cm}^{-1}$ [8], the transitions becoming allowed through Coriolis perturbation. $\quad \nu_{5}$ has apparently been observed in the Raman also at $1333 \mathrm{~cm}^{-1}[9]$.

No account was taken of the effect of centrifugal distortion in this analysis. Since only levels with low $J$ values were used in the analysis, the effect of this correction on the rotational constants was minimized. No systematic differences between the observed and calculated spectra were noticed until rather high $\boldsymbol{J}$ values were reached. In these regions of the absorption serious overlapping of transitions make the unique assignment of transitions to observed absorption peaks doubtful.

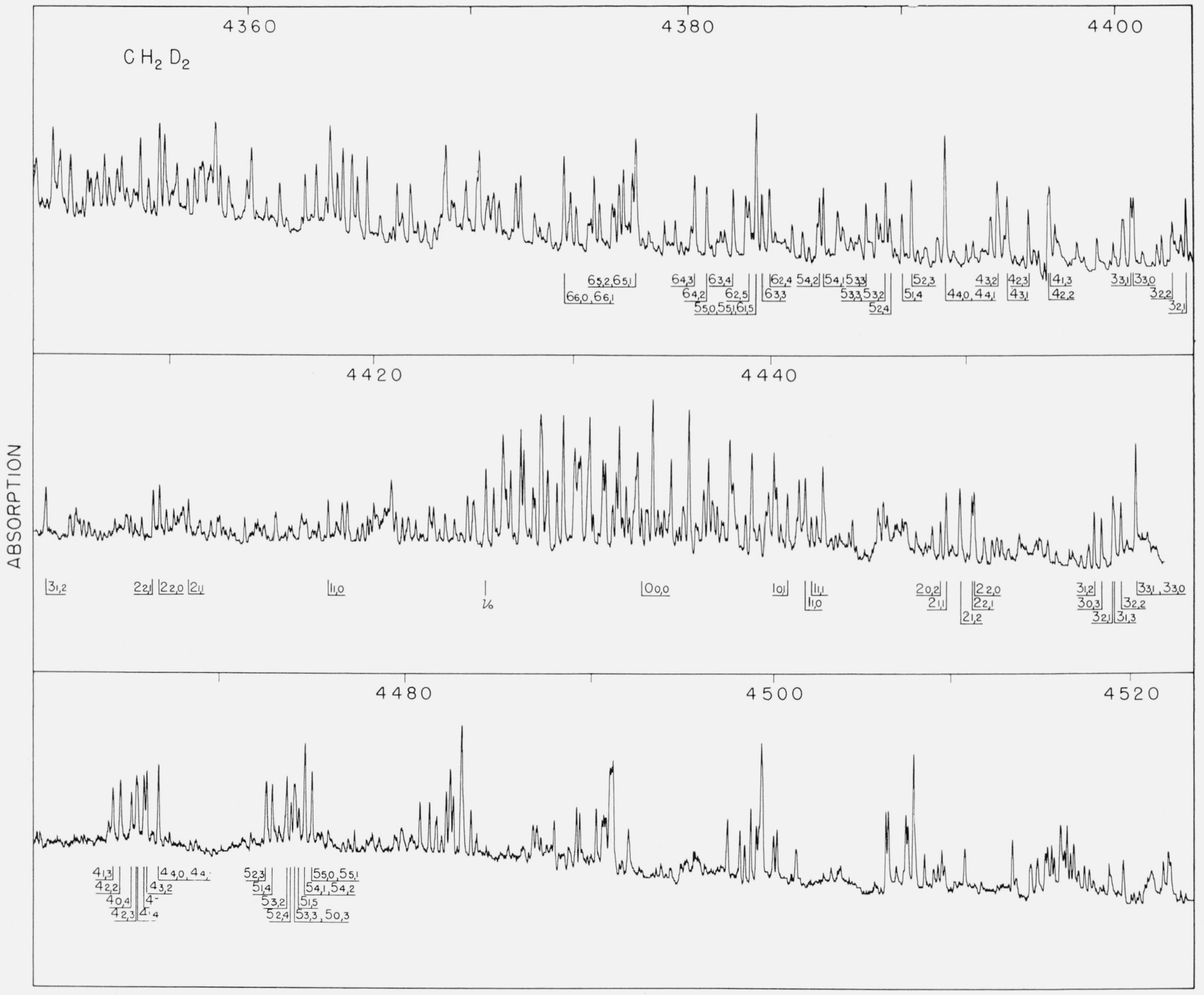

WAVE NUMBER, $\mathrm{cm}^{-1}$

Figure 2. The $C$-type band of $\mathrm{CH}_{2} \mathrm{D}_{2}$ at $4426 \mathrm{~cm}^{-1}$.

The identification given is the ground state designation of $J_{K_{-1}, K_{1}}$ for $R_{1,0}$ transitions to the high wave number side of the band origin, and for $P_{1}^{-}, 0$ transitions to the low wave number side of the band origin. 
Although no statistical analysis of the data was made, the excellent agreement between the observed and calculated $\Delta F_{2}$ values for the ground state, and the sensitivity of the calculated $\Delta F_{2}$ values to values of the rotational constants seem to indicate a probable error of the order of $\pm 0.002 \mathrm{~cm}^{-1}$ for each of the ground state constants. The agreement between the calculated and observed values of $\Delta F_{2}$ for the ground state may be seen in table 1 .

TABLE 1. Ground state $\Delta F_{2}^{\prime \prime}$

\begin{tabular}{|c|c|c|c|c|c|}
\hline \multicolumn{3}{|c|}{ A-type band } & \multicolumn{3}{|c|}{ C-type band } \\
\hline$\Delta F_{2}^{\prime \prime}$ & Calculated & Observed & $\Delta F_{2}^{\prime \prime}$ & Calculated & Observed \\
\hline $\begin{array}{l}0_{00}-2_{02} \\
1_{01}-3_{03} \\
1_{11}-3_{13} \\
1_{10}-3_{12}\end{array}$ & $\begin{array}{l}\mathrm{cm}^{-1} \\
19.513 \\
32.103 \\
31.547 \\
33.791\end{array}$ & $\begin{array}{r}c m^{-1} \\
19.55 \\
32.10 \\
31.55 \\
33.79\end{array}$ & $\begin{array}{l}0_{00}-2_{20} \\
1_{01}-3_{21} \\
1_{11}-3_{31} \\
1_{10}-3_{30}\end{array}$ & $\begin{array}{l}c m^{-1} \\
23.911 \\
37.524 \\
41.285 \\
40.862\end{array}$ & $\begin{array}{r}c m^{-1} \\
23.92 \\
37.53 \\
41.29 \\
40.88\end{array}$ \\
\hline $\begin{array}{l}202-4_{04} \\
2_{12}-4_{14} \\
2_{11}-4_{13} \\
2_{21}-4_{23} \\
2_{20}-4_{22}\end{array}$ & $\begin{array}{l}44.373 \\
43.976 \\
46.958 \\
45.754 \\
47.277\end{array}$ & $\begin{array}{l}44.38 \\
43.98 \\
46.96 \\
45.76 \\
47.25\end{array}$ & $\begin{array}{l}2_{02}-4_{22} \\
2_{12}-4_{32} \\
2_{11}-4_{31} \\
2_{21}-4_{41} \\
2_{20}-4_{40}\end{array}$ & $\begin{array}{l}51.644 \\
55.068 \\
53.908 \\
58.305 \\
58.165\end{array}$ & $\begin{array}{l}51.64 \\
55.06 \\
53.91 \\
58.27 \\
58.16\end{array}$ \\
\hline $\begin{array}{l}3_{03}-5_{05} \\
3_{13}-5_{15} \\
3_{12}-5_{14} \\
3_{22}-5_{24} \\
3_{21}-5_{23} \\
3_{31}-5_{33} \\
3_{30}-5_{32}\end{array}$ & $\begin{array}{l}56.501 \\
56.300 \\
59.715 \\
58.539 \\
60.967 \\
59.463 \\
60.141\end{array}$ & $\begin{array}{l}56.50 \\
56.28 \\
59.69 \\
58.54 \\
60.96 \\
59.47 \\
60.13\end{array}$ & $\begin{array}{l}3_{03}-5_{23} \\
3_{13}-5_{33} \\
3_{12}-5_{32} \\
3_{22}-5_{42} \\
3_{21}-5_{41} \\
3_{31}-5_{51} \\
3_{30}-5_{50}\end{array}$ & $\begin{array}{l}66.378 \\
69.201 \\
67.212 \\
71.733 \\
71.127 \\
75.463 \\
75.431\end{array}$ & $\begin{array}{l}66.37 \\
69.20 \\
69.23 \\
71.73 \\
71.12 \\
75.43 \\
75.43\end{array}$ \\
\hline $\begin{array}{l}4_{04}-606 \\
4_{14}-616 \\
4_{13}-615 \\
4_{23}-6_{25} \\
4_{22}-624 \\
4_{32}-6_{34} \\
4_{31}-633 \\
4_{41}-643 \\
4_{40}-642\end{array}$ & $\begin{array}{l}68.637 \\
68.559 \\
72.042 \\
71,147 \\
74.350 \\
72.585 \\
74.092 \\
72.848 \\
73.062\end{array}$ & $\begin{array}{l}68.61 \\
68.53 \\
71.98 \\
71.11 \\
74.33 \\
72.55 \\
74.07 \\
72.81 \\
73.02\end{array}$ & $\begin{array}{l}4_{04}-6_{24} \\
4_{14}-6_{34} \\
4_{13}-6_{33} \\
4_{23}-6_{43} \\
4_{222}-6_{42} \\
4_{32}-6_{52} \\
4_{31}-6_{51} \\
4_{41}-6_{61} \\
4_{40}-6_{60}\end{array}$ & $\begin{array}{l}81.651 \\
83.676 \\
81.042 \\
85.399 \\
83.950 \\
88.728 \\
88.532 \\
92.665 \\
92.660\end{array}$ & $\begin{array}{l}81.64 \\
83.63 \\
81.03 \\
85.38 \\
83.93 \\
88.65 \\
88.52 \\
92.56 \\
92.56\end{array}$ \\
\hline
\end{tabular}

TABLE 2. C-type band $\Delta F_{2}^{\prime}$

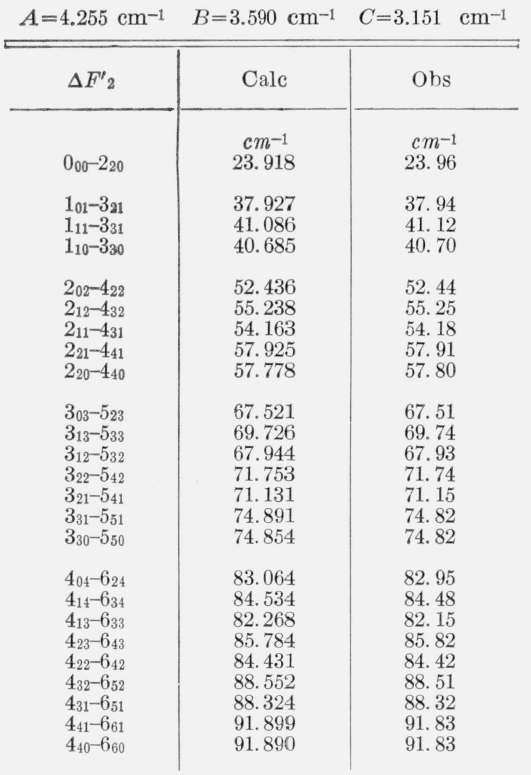

The constants for the excited states of these bands cannot be determined as precisely as those for the ground state with the available $\Delta F_{2}$ values, but $\pm 0.005 \mathrm{~cm}^{-1}$ would seem to be a generous estimate of the probable error in the constants for these states. The calculated and observed $\Delta F_{2}$ values for the excited states are compared in tables 2 and 3.

The constants determined for the three vibrational levels are given in table 4 . The band origins were determined from the best fit between the observed and calculated spectra for low $J$ values.

TABLE 3. A-type band $\Delta F_{2}^{\prime}$

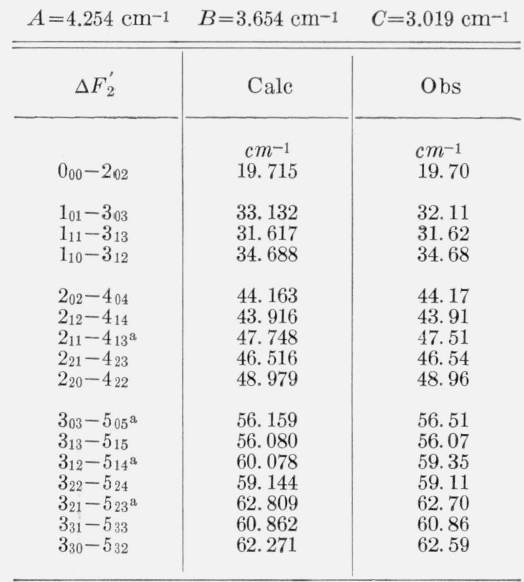

a Perturbed levels.

TABLE 4. Rotational and viōrational constants

\begin{tabular}{|c|c|c|c|}
\hline & Ground state & $\nu_{3}+\nu_{9}$ & $\nu_{3}+\nu_{6}$ \\
\hline $\begin{array}{l}A \\
B \\
B\end{array}$ & $\begin{array}{l}c m^{-1} \\
4.303 \\
3.504 \\
3.049 \\
-\cdots\end{array}$ & $\begin{array}{c}c m^{-1} \\
4.254 \\
3.654 \\
3.019 \\
\text { 2671. } 67\end{array}$ & $\begin{array}{r}c m^{-1} \\
4.255 \\
3.590 \\
3.151 \\
4425.61\end{array}$ \\
\hline
\end{tabular}

\section{References}

[1] Among the more recent high resolution analyses are those of: K. T. Hecht, J. Mol. Spectroscopy 5, 355 and 390 (1960). M. A. Thomas and H. L. Welsh, Can. J. Phys. 38, 1291 (1960).

J. Moret-Bailly, Cahiers de Phys. 130-131, 1 (1961).

R. A. Olafson, M. A. Thomas, and H. L. Welsh, Can. J. Phys. 39, 419 (1961).

H. C. Allen, Jr., and E. K. Plyler, J. Research NBS 63, 145 (1959).

E. H. Richardson, S. Brodersen, L. Krause, and H. L. Welsh, J. Mol. Spectroscopy 8, 406 (1962).

[2] H. C. Allen, Jr., Phil. Trans. Roy. Soc. London A 253, 335 (1961).

[3] N. Gailar and E. K. Plyler, J. Research NBS 45, 102 (1955).

[4] E. K. Plyler, L. R. Blaine, and E. D. Tidwell, ibid., 55, $279(1955)$

[5] C. H. Townes and A. L. Schawlow, Nicrowave Spectroscopy, McGraw-Hill, New York, (1955).

[6] J. K. Bragg and S. Golden, Phys. Rev.

[7] P. C. Cross, R. M. Hainer, and G. W. King, J. Chem. Phys. 12, 210 (1944).

[8] J. K. Wilmshurst and H. J. Bernstein, Can. J. Chem. 35, 226 (1957)

[9] G. E. MacWood and H. C. Urey, J. Chem. Phys, 4, 402 (1936).

(Paper 67A1-191) 